\title{
Designing Power Efficient Fibonacci Generator Using Different FPGA Families
}

\author{
Abhay Saxena $^{\# 1}$, Puneet Chandra Verma ${ }^{* 2}$, Chandrashekhar ${ }^{\# 3}$ Pragya Agarwal ${ }^{* 4}$, Apurva Omer ${ }^{\# 5}$ \\ 1,2,3,4,5 Department of Computer Science DSVV Haridwar, India \\ 1 abhaysaxena2009@gmail.com, ${ }^{2}$ cvermapuneet@gmail.com \\ ${ }^{3}$ shekharrockin1988@gmail.com, ${ }^{4}$ pragyaagarwal30@gmail.com, ${ }^{5}$ apurva.omer@dsvv.ac.in
}

\begin{abstract}
In consideration to wireless communication Fibonacci number is used to generate WPA and WPA2 (Wi-Fi Protected Access) key. Here, in our work we have designed green Fibonacci Generator under different FPGA families. Families we taken into consideration is Automotive Artix7, Artix7 and Kintex7.

First we have calculated power consumption of our designed at 2 Volt \& $1 \mathrm{GHz}$ frequency and change the capacitance from 5 to 30pf and found there is tiny changes in Artix7and kintex7 Family but got significant changes in Automotive Artix 7 around $16.79 \%$. Secondly we have calculated power consumption of our designed at $2 \mathrm{Volt} \& 10 \mathrm{GHz}$ frequency and change the capacitance from 5 to $30 \mathrm{pf}$, and found $41.09 \%, 13.95 \%$ and $38.06 \%$ change respectively for Automotive Artix7, Artix7 and Kintex7 FPGA families. Third we have worked with $3 \mathrm{~V}$ and $1 \mathrm{GHz}$ and got tiny changes with Artix7 and Kintex7 and found error value with Automotive Artix7. Lastly we have worked with $3 \mathrm{~V}$ and $10 \mathrm{GHz}$ and changed the capacitance from 5 to 30 pf, we got $4.7 \%$ and $30.10 \%$ significant reduction in power consumption for Artix7 and Kintex7 FPGA families but for Automotive Artix7 we again got error value.
\end{abstract}

Keyword - Artix7, kentix7, FPGA, Energy Efficient Design

\section{Introduction}

The Fibonacci numbers are the numbers in which every number is the sum of the preceding two numbers. This sequence is known as Fibonacci sequence. The first two numbers in the Fibonacci series are either 0 and 1 , or 1 and 1 , depending on the selected starting point of the series, and each succeeding number is the sum of the previous two numbers. Here we have designed our Fibonacci generator and test the power consumption in different FPGA families.

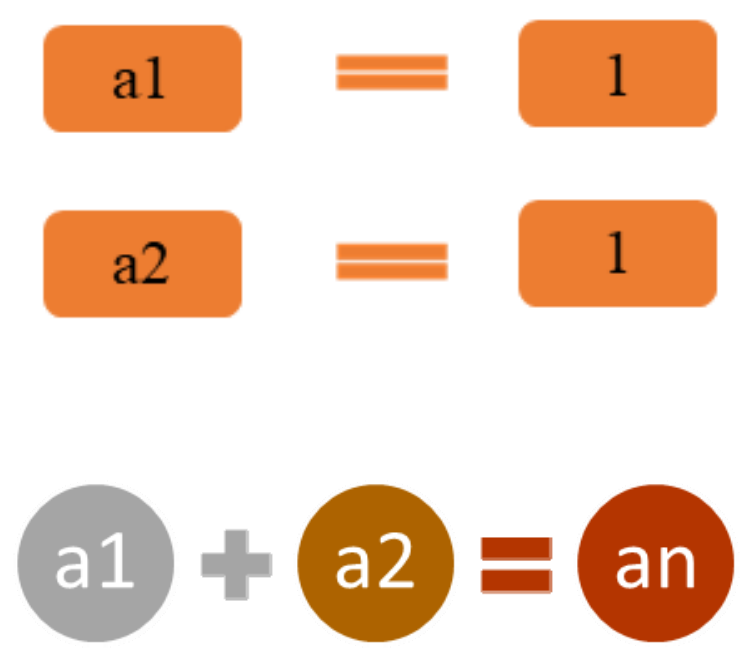

Fig:1 Idea of Fibonacci generator

In figure 1 we have shown that an is the next element of the series. That is, the current value is the sum of the previous two values. In figure 2 and figure 3 we have shown Top Level of Schematic of Fibonacci Generator and RTL Schematic of Fibonacci Generator respectively. 


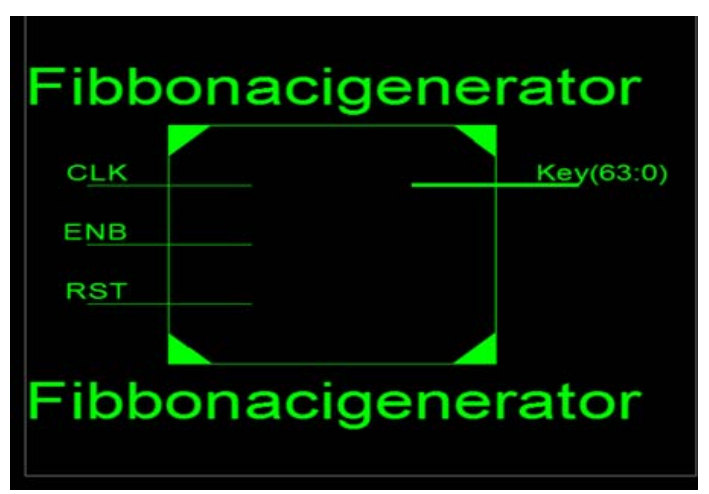

Figure 2: Top Level of Schematic of Fibonacci Generator

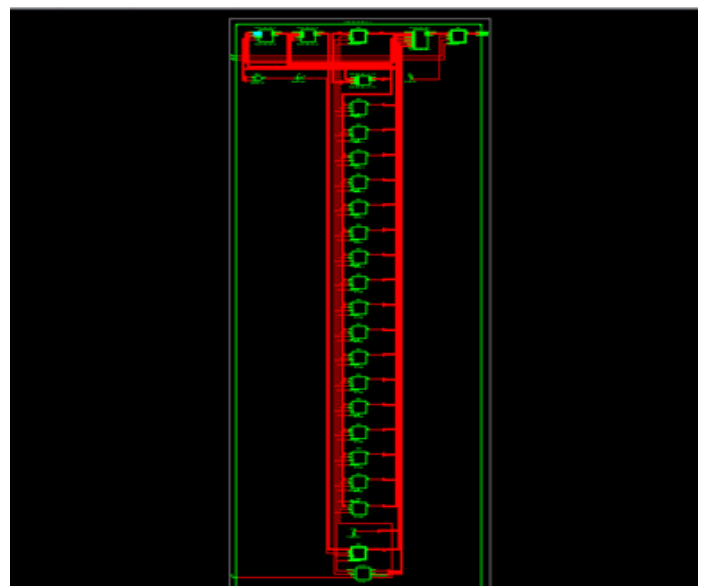

Figure 3: RTL Schematic of Fibonacci Generator

\section{Literature Work}

CRC is an error detection technique [1]. High Performance FIFO Design for Processor through Voltage Scaling Technique [2].HSTL IO Standards Based Processor Specific Green Counter[3].Capacitance Scaling Based Low Power Comparator Design on 28nm FPGA[4]. SSTL Based Energy Efficient FIFO Design for High Performance Processor of Portable Devices [5]. Energy Efficient CRC Design for Processor of Workstation, and Server using LVCMOS [6].Cyclic redundancy check is a basic requirement for speed-optimized computation [6]. Memory controller logic includes a CRC component configured to enable the CRC processes on the individual ranks [6]. Speed-optimized computation of cyclic redundancy check codes[7]. Cyclic Redundancy Check (CRC) False Detection Reduction in Communication Systems[8].In reference [9], researcher developed an instruction set architecture for programmable cyclic redundancy check (CRC) computation.Energy Efficient ALU Design Based On Voltage Scaling[10]

\section{RESULT}

TABLE I. Power consumption at $2 \mathrm{~V} \& 1 \mathrm{GHz}$

\begin{tabular}{|c|c|c|c|}
\hline capacitance(pf) & Automotive Artix7 & Artix7 & kentix7 \\
\hline 5 & 0.54 & 0.455 & 0.633 \\
\hline 10 & 0.584 & 0.457 & 0.635 \\
\hline 15 & 0.649 & 0.459 & 0.638 \\
\hline
\end{tabular}

In table 1, power consumption of Fibonacci Generator is shown at $1 \mathrm{GHz}$ frequency and 2V. In our table total power consumption is sum of I/O Power \& Leakage Power. There is drop by $15.74 \%$ as we move from Automotive Artix7 to Artix7 and there in increment by $39.12 \%$ as we have moved from Artix7 to Kentix7 at 5pF. There is drop by $21.74 \%$ as we move from Automotive Artix7 to Artix7 and there in increment by 38.94\% as we move from Artix7 to Kentix7 at $15 \mathrm{pF}$. There is drop by $29.27 \%$ as we move from Automotive Artix7 to Artix7 and there is increment by $38.99 \%$ as we have moved from Artix7 to Kentix7 at 30pF. The above table data is shown by fig.4. 


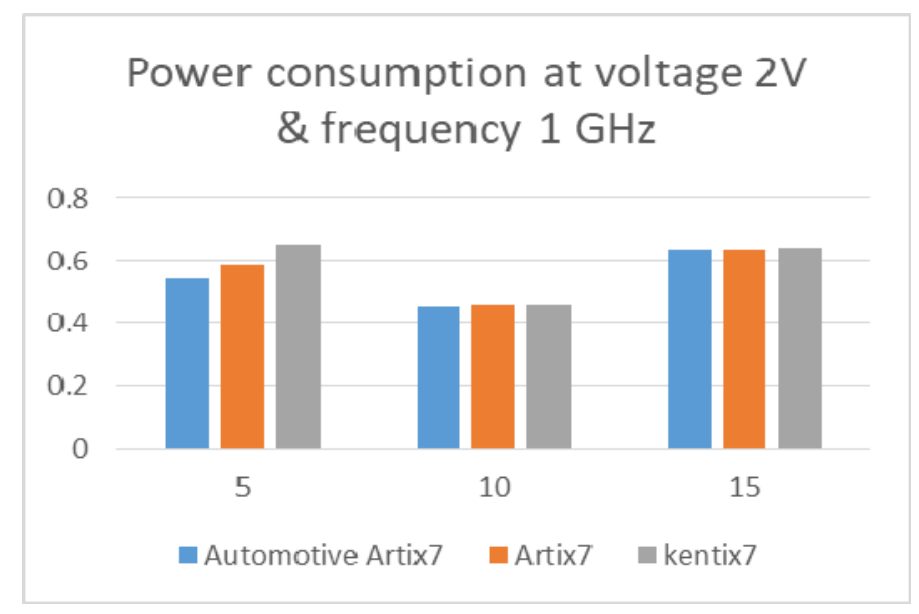

Fig.4 Power consumption at 2Volt \& $1 \mathrm{GHz}$

TABLE II. Total load at 2 Volt \& $10 \mathrm{GHz}$

\begin{tabular}{|c|c|c|c|}
\hline capacitance(pf) & Automotive Artix7 & Artix7 & kentix7 \\
\hline 5 & 1.587 & 0.937 & 1.783 \\
\hline 10 & 2.028 & 0.998 & 2.221 \\
\hline 15 & 2.694 & 1.089 & 2.879 \\
\hline
\end{tabular}

In table 2, power consumption of Fibonacci Generator is shown at $10 \mathrm{GHz}$ frequency and Voltage 2V. In our work we have found that there is drop by $40.95 \%$ as we have moved from Automotive Artix7 to Artix7 and there in increment by $90.28 \%$ as we switch from Artix7 to Kentix7 at $5 \mathrm{pF}$. There is drop by $50.78 \%$ as we go from Automotive Artix7 to Artix7 and there in increment by $122.54 \%$ as we shift from Artix7 to Kentix7 at $10 \mathrm{pF}$. There is drop by $59.57 \%$ as we move from Automotive Artix7 to Artix7 and there in increment by $164.3 \%$ as we changed from Artix7 to Kentix7 at 15pF. The above table data is also shown by Figure 5.

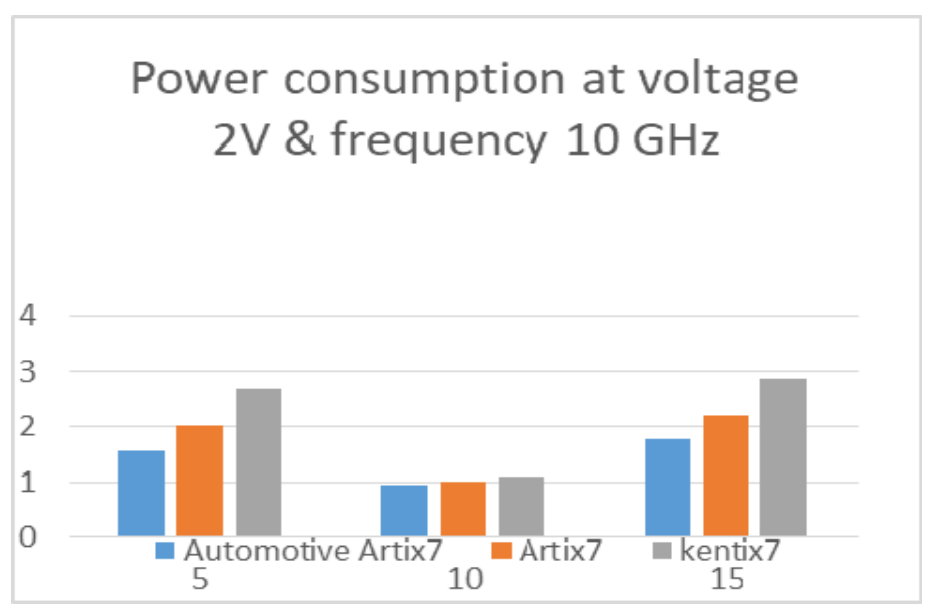

Fig.5 Power consumption at 2Volt \& $10 \mathrm{GHz}$

TABLE III. Total load at voltage 3V \& frequency $1 \mathrm{GHz}$

\begin{tabular}{|c|c|c|c|}
\hline capacitance(pf) & Automotive Artix7 & Artix7 & kentix7 \\
\hline 5 & 241.12 & 9.876 & 16.667 \\
\hline 10 & - & 9.881 & 16.674 \\
\hline 15 & - & 9.889 & 16.686 \\
\hline
\end{tabular}

In table 3, power consumption of Fibonacci Generator is shown at $1 \mathrm{GHz}$ frequency and Voltage 3V. In our work we have found that there is lot of power consumption in Automotive Artix7 so we got error value for higher Capacitance. There is increment by $68.76 \%$ as we have moved from Artix7 to Kentix7 at $5 \mathrm{pF}$. There is increment by $68.74 \%$ as we shift from Artix7 to Kentix7 at $10 \mathrm{pF}$. There is increment by $68.73 \%$ as we change from Artix7 to Kentix7 at 15pF. The above table data is also shown by Figure 6 . 


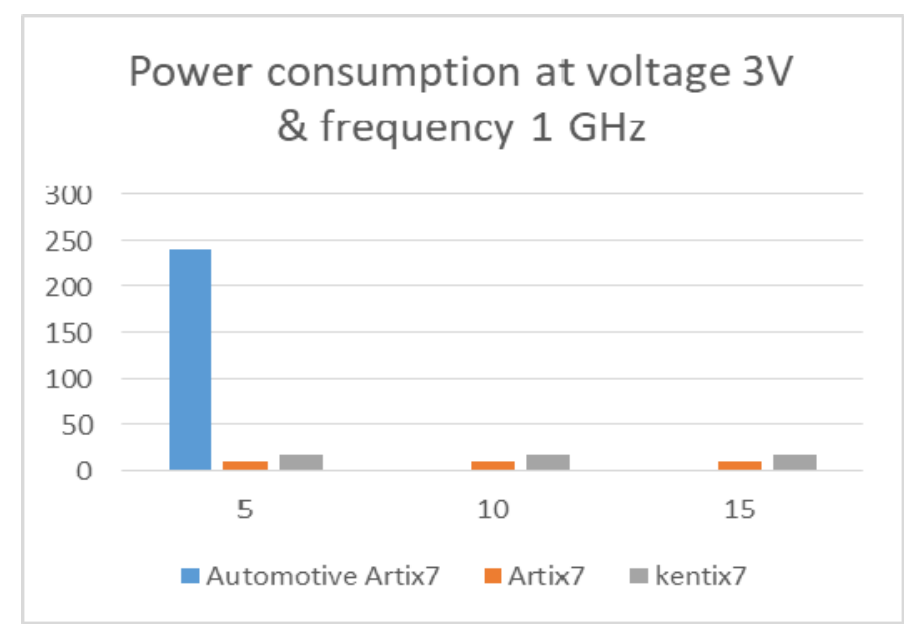

Fig.6 Power consumption at 3Volt \& $1 \mathrm{GHz}$

TABLE IV. Total load at voltage 3V \& frequency $10 \mathrm{GHz}$

\begin{tabular}{|c|c|c|c|}
\hline capacitance(pf) & Automotive Artix7 & Artix7 & kentix7 \\
\hline 5 & 241.117 & 12.804 & 23.36 \\
\hline 10 & - & 13.053 & 26.119 \\
\hline 15 & - & 13.44 & 33.423 \\
\hline & & 4.7 & 30.10 \\
\hline
\end{tabular}

In table 4, power consumption of Fibonacci Generator is shown at $10 \mathrm{GHz}$ and 3Volt. In our work we have found that there is lot of power consumption in Automotive Artix7 so we got error value recorded for higher Capacitance. There is increment by $82.44 \%$ as we go from Artix7 to Kentix7 at $5 p F$. There is increment by $100.09 \%$ as we shift from Artix7 to Kentix7 at 10pF. There is increment by $148.68 \%$ as we move from Artix7 to Kentix7 at $15 \mathrm{pF}$. The above table data is also shown by Figure 7.

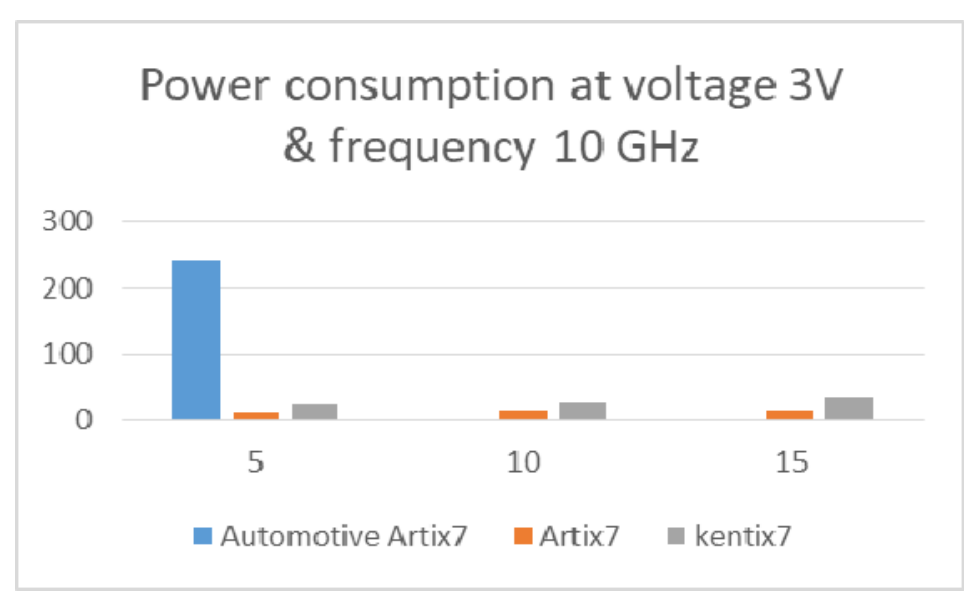

Fig.7 Power consumption at 2Volt \& $10 \mathrm{GHz}$

\section{CONCLUSION}

In our paper we have designed Power Efficient Fibonacci Generator using different FPGA families using VHDL language. Here we have applied capacitance scaling techniques for calculating total power consumption. As we know that Clock Power \& Signal Power are independent of capacitance scaling and I/O Power \& Leakage Power is varying with changing capacitance, so in our work, total power consumption is sum of I/O Power \& Leakage Power. First we have calculated power consumption of our designed at $2 \mathrm{Volt} \& 1 \mathrm{GHz}$ frequency and found the significant power consumption in Automotive Artix7 FPGA family. Secondly we have calculated power consumption of our designed at 2Volt \& $10 \mathrm{GHz}$ frequency and found $41.09 \%$ power reduction in Automotive Artix7 which is highest among the other FPGA families. In third, we have worked with 3Volt and frequency $1 \mathrm{GHz}$ and got error value for Automotive Artix7 FPGA. In last, we have worked with 3Volt \& $10 \mathrm{GHz}$ and found $30.10 \%$ reduction in power consumption in kentix7 family which is highest among the other FPGA family . 


\section{FUTURE SCOPE}

In this work, CRC Design is implemented on 28nm Kintex-7 FPGA family, but we have scope to redesign our CRC with different FPGA family like Virtex 7,Virtex 6, Virtex 5 or we can also apply different- different techniques for calculating total power consumption.

\section{REFERENCES}

[1] CRC polynomial, http://ghsi.de/CRC/index.php, Last Visited on: 04 April 2016

[2] A Saxena, A Bhatt, P Gautam, P Verma, C Patel,”High Performance FIFO Design for Processor through Voltage Scaling Technique” In Indian Journal of Science and Technology Vol 9(45), DOI: 10.17485/ijst/2016/v9i45/106916, December 2016.

[3] A.Saxena, A.Bhatt B.Pandey, P.Tripathi“HSTL IO Standards Based Processor Specific Green Counter.”In International Journal of Control and Automation, Vol. 9, No. 7, (2016), pp. 331-342.

[4] A Saxena, S Gaidhani, A Pant, C Patel "Capacitance Scaling Based Low Power Comparator Design on 28nm FPGA" in International Journal of Computer Trends and Technology (IJCTT) - Volume X Issue Y- Month 2015

[5] A Saxena, S Sharma,P Agarwal, C Patel "SSTL Based Energy Efficient FIFO Designfor High Performance Processor ofPortable Devices " in International Journal of Engineering and Technology (IJET)Vol 9 No 2 Apr-May 2017DOI: 10.21817/ijet/2017/v9i2/170902113.

[6] A Saxena,C Patel,M.Khan "Energy Efficient CRC Design for Processor ofWorkstation, and Server using LVCMOS " in Indian Journal of Science and Technology, Vol 10(4), DOI: 10.17485/ijst/2017/v10i4/110890, January 2017.

[7] C. D. Kirkpatrick, "Speed-optimized computation of cyclic redundancy check codes." U.S. Patent No. 8,R.

[8] Subashchandrabose, et al. "Rank-specific cyclic redundancy check." U.S. Patent No. 8,745,464. 3 Jun. 2014.468,439. 18 Jun. 2013.

[9] R. Cohen, and A. Kleinerman. "Cyclic Redundancy Check (CRC) False Detection Reduction in Communication Systems" U.S. Patent No. 20,160,026,523. 28 Jan. 2016.

[10] V. Gopal, et al. "Instruction-set architecture for programmable cyclic redundancy check (CRC) computations." U.S. Patent No. 8,732,548. 20 May 2014

[11] A Saxena,C Patel,M.Khan "Energy Efficient ALU Design Based On Voltage Scaling” in Gyancity Journal of Electronics and Computer Science,Vol.1, No.1, pp.29-33, September 2016ISSN: 2446-2918 DOI: 10.21058/gjecs.2016.11006. 